\title{
Alcohol, Central Nervous System Injury, and Time to Death in Fatal Motor Vehicle Crashes
}

\author{
Brian J. Zink, Ronald F. Maio, and Bonny Chen
}

\begin{abstract}
Objectives: Motor vehicle crash (MVC) studies have found that alcohol (ALC) is associated with increased mortality and decreased time to death (TTD). Clinical and experimental data suggest that ALC potentiates central nervous system injury (CNSI). We hypothesize that ALC-intoxicated, MVC fatalities with CNSI are more likely to die in the immediate postinjury period than are sober victims with CNSI. Methods: Design-A retrospective cohort of 401 MVC fatalities from four Michigan counties for the time period 1985 to 1991 was studied. Measurements-Medical examiner records were reviewed to determine age, blood alcohol concentration (BAC), and TTD. Injury severity was calculated with the Abbreviated Injury Scale (1985 version). Anatomical profile scores and $G$ scores were also calculated and used to identify CNSI subjects. Analysis- $\chi^{2}$ and Student's $t$ test were used, and odds ratios with 0.95 confidence intervals (Cls) were calculated. Results: ALC( + ) cases (BAC $\geq 100 \mathrm{mg} / \mathrm{dl})(n=99)$ were significantly younger and more frequently had $T T D<1 \mathrm{hr}$ than ALC $(-)$ cases $(n=233)$ : odds ratio 1.62 [0.95 Cl (1.02 to 2.56)]. Overall, CNSI cases $(n=297)$ were significantly younger and had fewer thoracic injuries, but did not have significantly shorter TTD, compared with non-CNSI cases. However, ALC( + ) CNSI cases $(n=77)$ were over twice as likely to have TTD $<1 \mathrm{hr}$ fodds ratio 2.04 [0.95 $\mathrm{Cl}$ (1.13 to 3.70)]\}. For ALC(+) isolated CNSI cases, the odds ratio for TTD $<1$ hr, compared with nonisolated CNSI cases was 8.25 (0.95; $\mathrm{Cl} 0.66$ to 102.5). Injury Severity Score, anatomical profile, and $G$ scores were not significantly different for ALC(+) CNSI cases, compared with ALC(-) CNSI cases, whether isolated or nonisolated. Concluslons: These data suggest that alcohol intoxication is associated with increased frequency of early death in MVC victims with CNSI, despite there being no detectable difference in anatomical injury scoring.

Key Words: Alcohol, Brain Injury, Motor Vehicle Crashes, Trauma.
\end{abstract}

A LCOHOL USE predisposes to traumatic injury. Numerous studies have found that 25 to $50 \%$ of injured patients who present to the emergency department (ED) are alcohol intoxicated. ${ }^{1}$ Motor vehicle crashes (MVCs) constitute a major part of the problem of alcohol-related injury. Alcohol intoxication is found in 50\% of traffic fatalities and in approximately one-third of nonfatally injured MVC victims. ${ }^{2-5}$ In their landmark study, Waller et al. ${ }^{5}$ found that alcohol intoxication had a potentiating effect on injury in MVC victims.

Central nervous system (CNS) injury (CNSI) accounts for much of the mortality and morbidity seen in both intoxicated and sober MVC victims. CNSI is found in up to

From the Department of Surgery, Section of Emergency Medicine, University of Michigan, Ann Arbor, Michigan.

Received for publication April 24, 1996; accepted August 28, 1996

Reprint requests: Brian J. Zink, M.D., Department of Surgery, Section of Emergency Medicine, TC B1354, University of Michigan, 1500 East Medical Center Drive, Ann Arbor, MI 48109-0303.

Copyright $\mathbb{0} 1996$ by The Research Society on Alcoholism.
$60 \%$ of patients who die or are seriously injured in MVCs. ${ }^{6,7}$ Both laboratory and clinical evidence exist to support the concept that alcohol worsens CNSI. ${ }^{8-15}$ The reasons for this have not been defined, but laboratory studies support a mechanism that is active within minutes to hours after injury. In a porcine fluid-percussion traumatic brain injury (TBI) model, alcohol has been found to prolong postinjury apnea and impair respiratory drive in the immediate postinjury period. ${ }^{16}$

Three previous studies have examined the relationship between alcohol use and time to death (TTD) in MVC fatalities. ${ }^{17-19}$ All have found that alcohol-intoxicated MVC victims were significantly more likely to die at the scene, or in the first hour after injury, than were sober victims. These studies did not control for crash characteristics or report injury patterns or types. We undertook this study to examine the effects of alcohol intoxication on TTD in a cohort of MVC fatalities with CNSI. We hypothesized that alcoholintoxicated, brain-injured MVC fatalities with CNSI were more likely to die in the immediate postinjury period than were sober victims with CNSI.

\section{METHODS}

\section{Design}

The study population was a retrospective cohort of $401 \mathrm{MVC}$ fatalities, age 13 years or older, from four counties in the state of Michigan for the years 1986 to 1991 . This database was previously used to analyze geographic variation in preventable deaths from MVCs. Three counties were rural and one was nonrural, with $32 \%$ of total subjects coming from the rural counties. All rural MVC fatalities were included in the analysis, and 51 eligible cases per year were randomly selected in the nonrural county. ${ }^{20}$ MVC victims who died within 30 days and had complete autopsies performed with autopsy records and medical examiner reports available for review were included in the study.

\section{Measurements}

Age, sex, date, time, and location of the MVC, TTD, and blood alcohol concentration (BAC) were recorded. Injury severity was determined from autopsy records using the Abbreviated Injury Scale (1985 version) and Injury Severity Score (ISS) as previously described. ${ }^{20,21}$ The Anatomical Profile (AP) score was also calculated. AP scoring, first described by Copes et al., ${ }^{21}$ expands upon conventional ISS scoring by taking into account multiple injuries within body regions. The AP score is calculated by summing the three highest scoring major injuries within each of the three ISS components $(A, B$, and $C$, where APA = brain, head, and spinal cord; APB = chest; and APC = abdomen and pelvis). The G score, like the ISS, is a summary score, but is derived from logistic regression that relates the AP component values to survival probability. A lower $G$ score indicates more severe injury. ${ }^{20,21}$ Subjects with brain injury or spinal cord 
injury as identified by the APA component score were termed CNSI subjects. A decision rule was constructed to define further CNS injuries that were likely to be fatal. Documented brain injury and/or spinal cord injury at the level of the second cervical vertebrae or higher was automatically considered CNSI. Spinal cord injury cases where the lesion was below the second cervical vertebrae were reviewed to determine the likelihood that spinal cord injury contributed to death. Cases with spinal cord injury below the second cervical vertebrae whose APA score was greater than the APB or APC score were classified as CNSI cases. Spinal cord injury cases with APA scores less than APB or APC scores were not considered as CNSI for the purposes of this analysis. Because the focus of the investigation was on mortality after injury, this decision rule allowed for identification of serious brain and upper spinal cord injuries, but excludes lower spinal cord injuries that would not be likely to cause early death in the absence of other injuries. Subjects were considered to have isolated CNSI if they had positive APA scores and had APB and APC scores of 0 .

TTD was defined as the elapsed time from the MVC until the recorded TTD in the medical record or autopsy record. BACs were taken from the medical examiner record. Samples were obtained at the hospital or upon autopsy. An alcohol level $\geq 100 \mathrm{mg} / \mathrm{dl}$ was used to define an alcoholpositive $[\mathrm{ALC}(+)]$ subject. This level is consistently used to denote alcohol intoxication in previous studies..$^{2-4,13,14}$ In addition, this is a widely accepted legal level of alcohol intoxication. Alcohol-negative [ALC $(-)$ ] subjects had alcohol levels $<100 \mathrm{mg} / \mathrm{dl}$.

\section{Data Analysis}

$\mathrm{ALC}(+)$ and $\mathrm{ALC}(-)$ groups were compared for the following variables: age, ISS, AP score, G score, and minutes to death. Subjects who were not tested for alcohol were compared with tested subjects. To identify subjects who died at the crash scene or in the prehospital or early hospital course of their care, subjects were grouped and analyzed with TTD $\leq 1 \mathrm{hr}$ and TTD $>1 \mathrm{hr}$. CNSI subjects were classified as ALC $(+)$ or $\mathrm{ALC}(-)$, and comparisons were performed for the following variables: age, ISS, AP and G scores, minutes to death, and TTD $\leq 1$ or $>1 \mathrm{hr}$. Subjects were also grouped as CNSI-positive or -negative, and these variables were compared. The $\chi^{2}$ test was used to compare categorical data between two groups, and Student's two-tailed $t$ test was used for interval data. Fischer's exact test was used for categorical data with less than five observations in each group. Odds ratios with 0.95 confidence intervals were calculated for TTD analyses.

\section{RESULTS}

Cases (449) were available for review in the database. Forty-eight cases with age $<13$ years were excluded, leaving $401 \mathrm{MVC}$ fatalities available for analysis. BAC was measured in 332 cases. When those cases who had BACs tested were compared with those who were not tested, no significant differences were found with regard to age, ISS score, AP score, or G score. Elapsed minutes to death was significantly longer in those cases where BAC was not tested $(311 \pm 1775 \mathrm{~min}$ for subjects tested for alcohol vs. $2216 \pm 6100 \mathrm{~min}$ for those not tested; $p<0.001)$. The percentage of cases with time to death $\leq 1 \mathrm{hr}$ was lower in nontested subjects, but the difference was not statistically significant $(65 \%$ in tested subjects and $59 \%$ for those not tested; $p=0.349$ ).

The average $\mathrm{BAC}$ for $\mathrm{ALC}(+)$ subjects was $200 \pm 74$ $\mathrm{mg} / \mathrm{dl}$, with a range of $100 \mathrm{mg} / \mathrm{dl}$ to $440 \mathrm{mg} / \mathrm{dl}$. The ALC(-) group had an average of $9 \pm 21 \mathrm{mg} / \mathrm{dl}$ and a range of 0 to $90 \mathrm{mg} / \mathrm{dl}$. When ALC( + ) subjects were compared with
Table 1. Comparison of $A L C(+)$ and $A L C(-)$ Subjects for Age, Injury Component Scores, and Minutes to Death

\begin{tabular}{lccc}
\hline \multicolumn{1}{c}{ Parameter } & ALC(-) & ALC(+) & $p=^{*}$ \\
\hline$n=$ & 233 & 99 & \\
Age $(\mathrm{yr})$ & $39(21)$ & $33(13)$ & 0.039 \\
ISS & $52(22)$ & $55(22)$ & 0.305 \\
G score & $-1.5(2.7)$ & $-1.8(28)$ & 0.350 \\
APA score & $5.4(4.4)$ & $5.7(4.6)$ & 0.585 \\
APB score & $7.1(4.5)$ & $7.2(4.7)$ & 0.840 \\
APC score & $1.8(2.3)$ & $2.3(2.4)$ & 0.117 \\
Minutes to death & $388(2088)$ & $132(534)$ & 0.231 \\
\hline
\end{tabular}

Numbers are means with standard deviations in parentheses.

* Student's two-tailed $t$ test.

Table 2. Comparison of CNSI Subjects and Non-CNSI Subjects for Age, Injury Component Scores, and TTD

\begin{tabular}{lccc}
\hline \multicolumn{1}{c}{ Parameter } & CNSI $(-)$ & CNSI $(+)$ & $p=^{*}$ \\
\hline$n=$ & 104 & 297 & \\
Age $(\mathrm{yr})$ & $44(22)$ & $36(18)$ & $<0.001$ \\
ISS & $50(25)$ & $53(22)$ & 0.22 \\
G score & $0.01(2.0)$ & $-2.1(2.8)$ & $<0.001$ \\
APB score & $9.0(4)$ & $6.3(4.5)$ & $<0.001$ \\
APC score & $2.2(2.3)$ & $1.9(2.3)$ & 0.20 \\
Minutes to death & $1142(4856)$ & $463(2109)$ & 0.052 \\
\%TTD $\leq 1 \mathrm{hr}$ & 62.5 & 65 & 0.55 \\
\hline
\end{tabular}

Numbers are means with standard deviations in parentheses.

- Student's two-tailed $t$ test, except for TTD analysis where Pearson's $\chi^{2}$ test was used.

$\mathrm{ALC}(-)$ subjects, ALC $(+)$ subjects were significantly younger and more frequently died in the first hour. Seventy-three percent of ALC $(+)$ subjects died in the first hour, compared with $62 \%$ of ALC $(-)$ subjects $(p=0.066$, Pearson's $\left.\chi^{2}\right)$. The odds ratio for dying within $1 \mathrm{hr}$ for ALC(+) subjects was 1.62 ( 0.95 confidence interval 1.02 to 2.56$)$. No significant differences were found between $\mathrm{ALC}(+)$ and ALC $(-)$ subjects in ISS, AP scores, or G scores. ALC(+) subjects had lower average minutes to death, but the difference was not statistically significant (Table 1).

Two hundred ninety-seven of 401 subjects (74\%) had CNSI, and 42 had isolated CNSI. Forty-four subjects had spinal cord injury. Ten of these subjects had APB $>$ APA scores, and using the decision rule were not classified as CNSI because the spinal cord injury was not considered to be lethal. None of these 10 cases had cord lesions above the sixth cervical level. When subjects with CNSI were compared with subjects without CNSI, CNSI subjects were significantly younger, and had significantly lower thoracic injury (APB) scores, G scores, and average minutes to death (Table 2). However, CNSI subjects were not more likely to die in the first hour after injury than non-CNSI subjects. Sixty-five percent of subjects with CNSI died within $1 \mathrm{hr}$, compared with $62.5 \%$ of non-CNSI subjects.

ALC(+) subjects with CNSI were significantly more likely to have TTD $\leq 1 \mathrm{hr}$ than ALC(-) subjects with CNSI. The difference was more pronounced for isolated CNSI than for nonisolated CNSI (Tables 3 and 4). For ALC( + ) subjects with CNSI, the overall odds ratio for TTD $\leq 1 \mathrm{hr}$ was 1.96 [0.95 confidence interval (1.03 to 3.41)]. For $\mathrm{ALC}(+)$ subjects with isolated CNSI, the odds 
Table 3. Comparison of $A L C(+)$ and $A L C(-)$ Subjects with CNSI for TTD Within $1 \mathrm{Hr}$

\begin{tabular}{ccccc}
\hline & TTD $\leq 1 \mathrm{hr}^{*}$ & TTD $>1 \mathrm{hr}$ & Total & $\% \mathrm{TTD} \leq 1 \mathrm{hr}$ \\
\hline ALC(-) & 106 & 68 & 174 & 77 \\
ALC(+) & 58 & 19 & & 75 \\
Total & 164 & 87 & 221 & \\
\hline
\end{tabular}

Odds ratio $=1.96[0.95$ confidence interval $(1.03-3.41)]$.

${ }^{\star} p=0.027$ Pearson's $\chi^{2}$.

Table 4. Comparison of $\mathrm{ALC}(+)$ and $\mathrm{ALC}(-)$ Subjects with Isolated CNSI for TTD Within $1 \mathrm{Hr}$

\begin{tabular}{|c|c|c|c|c|}
\hline & $\mathrm{TTD} \leq 1 \mathrm{hr}^{*}$ & $\pi \mathrm{TD}>1 \mathrm{hr}$ & Total & $\% \mathrm{TTD} \leq 1 \mathrm{hr}$ \\
\hline $\mathrm{ALG}(-)$ & 12 & 9 & 21 & 57 \\
\hline $\mathrm{ALC}(+)$ & 11 & 1 & 12 & 92 \\
\hline Total & 23 & 10 & 33 & \\
\hline
\end{tabular}

Odds ratio $=8.25[0.95$ confidence interval $(0.66-102.5)]$.

$p=0.054$ Fisher's Exact test (two-tailed).

Table 5. Comparison of ALC(+) CNSI Subjects and ALC $(-)$ CNSI Subjects for Age and Injury Component Scores, and Minutes to Death

\begin{tabular}{lccl}
\hline \multicolumn{1}{c}{ Parameter } & ALC(-) CNSI & ALC(+) CNSI & $p={ }^{*}$ \\
\hline$n=$ & 174 & 77 & \\
Age (yr) & $36(20)$ & $33(12)$ & 0.16 \\
ISS & $53(21)$ & $55(22)$ & 0.54 \\
G score & $-2.06(2.7)$ & $-2.2(2.8)$ & 0.63 \\
APA score & $7.1(3.7)$ & $7.3(3.9)$ & 0.75 \\
APB score & $6.4(4.4)$ & $6.5(4.8)$ & 0.93 \\
APC score & $1.9(2.4)$ & $2.1(2.4)$ & 0.47 \\
Minutes to death & $299(1495)$ & $84(334)$ & 0.21 \\
\hline
\end{tabular}

Numbers are means with standard deviations in parentheses.

* Student's two-tailed $t$ test.

ratio for TTD $\leq 1 \mathrm{hr}$ was 8.25 [0.95 confidence interval (0.66 to 102.5)]. The average minutes to death was not significantly greater in ALC $(+)$ subjects with isolated CNSI or nonisolated CNSI. Age, ISS scores, and AP scores were not significantly different between $\mathrm{ALC}(+)$ and $\mathrm{ALC}(-)$ subjects who had CNSI (Table 5).

\section{DISCUSSION}

Data from this retrospective analysis of regional MVC fatalities indicate that alcohol intoxication is associated with an increased frequency of early death, particularly when CNSI is present. This association between alcohol intoxication and early death emerged even though injury severity and AP scoring did not detect any significant differences between $\mathrm{ALC}(+)$ and $\mathrm{ALC}(-)$ subjects who sustained CNSI. This raises some interesting issues regarding the potential mechanism(s) for such a finding, as well as implications for prevention and treatment of CNSI in alcohol-intoxicated trauma victims.

Previous studies have discovered a potentiating effect of alcohol on injury and have found that alcohol-intoxicated MVC victims are more likely to die in the first hour after injury. Waller et al. ${ }^{5}$ published the first large, controlled analysis of the effect of alcohol intoxication in MVCs. When controlling for potentially confounding variablessuch as accident type and speed, vehicle weight, amount of vehicle deformation, seat belt use and age-alcohol intoxication was associated with a 2 -fold increase in death or serious injury. ${ }^{5}$ Using data from the Fatal Accident Reporting System (FARS), Evans and Frick ${ }^{3}$ also used an approach that controlled for accident severity and also found that fatality risk was 2-fold in alcohol-intoxicated MVC victims. Our data support this concept of a potentiating effect for alcohol, particularly in the early postinjury period. Other studies that have focused on the hospital course of the trauma victim without controlling for crash characteristics or mechanism of trauma have not demonstrated a potentiating effect of alcohol on injury. ${ }^{22-24}$

TTD may be an important marker in assessing the temporal course and nature of alcohol effects after injury. Three previous studies have found that alcohol is associated with decreased TTD after MVCs. Stewart, ${ }^{18}$ using methods similar to Waller et al. that controlled for vehicle deformation, speed, seat belt use, and age, found that a much higher proportion of subjects with $\mathrm{BAC} \geq 100 \mathrm{mg} / \mathrm{dl}$ died in the first hour after the crash when compared with subjects who had $\mathrm{BAC}=0$. Fell and Hertz ${ }^{17}$ using FARS data, found that the proportion of fatalities occurring in the first $30 \mathrm{~min}$ was significantly higher for all age and sex categories when the BAC was $>100 \mathrm{mg} / \mathrm{dl} .{ }^{17}$ An apparent dose-dependent effect was observed, with the proportion of drivers dying with 30 min, thus increasing in an almost linear manner with BAC. ${ }^{17}$ Dischinger et al. ${ }^{19}$ studied vehicular fatalities in Maryland in 1985 and found that victims who were alcohol-intoxicated were significantly more likely to die at the scene or be dead on arrival at a hospital than nonintoxicated victims. None of these studies reported injury patterns or looked specifically at brain injury. Our study agrees with the findings of these three prior TTD studies and provides additional data on anatomical injury.

A central question in interpreting the data from our study is: How can alcohol increase the frequency of early death in CNSI subjects without increasing anatomical injury scores? A small number of clinical studies have examined the effects of alcohol on outcome in cases of TBI. 
Kraus et al. ${ }^{13}$ found a correlation between increased BAC on presentation and neurological impairment and longer hospital stays in TBI patients. However, an inverse correlation existed for injury severity and hospital mortality. This study had potential selection bias introduced by low rates of BAC testing in TBI patients and inadequate reporting on prehospital deaths. Another study found no difference in mortality, but an increased need for early endotracheal intubation, ventilatory assistance, and intracranial pressure monitoring in alcohol-intoxicated patients with blunt TBI. ${ }^{14}$ Rönty et al. ${ }^{15}$ compared alcoholic and nonalcoholic patients with mild to moderate TBI, and found significantly larger initial brain lesion volumes and signs of brain abnormality on head CT scans in alcoholic subjects. Alcoholic patients had also reduced cerebral blood flow at the site of injury in the acute stage. Ability to return to work was markedly lower at 1 year in the alcoholic group. ${ }^{15}$

Laboratory studies have also found that alcohol potentiates CNSI. Four investigations, each using a different species and model of experimental TBI, have found that ethanol leads to larger lesion volumes, increased neuropathological changes, increased neurological impairment, and higher mortality. ${ }^{8-11}$ Two studies have looked at effects of alcohol in spinal cord injury, with both showing increased neurological damage in alcohol-treated animals. ${ }^{25,26}$ These animal studies do not provide a great deal of insight into the possible mechanism for alcohol potentiation of TBI, but suggest that postinjury activation of excitatory amino acids and free radicals may play a role., ${ }^{9,11,25,26}$

Our previous studies, using a porcine fluid-percussion TBI model, have found that ethanol-treated animals (ethanol levels $=120$ to $180 \mathrm{mg} / \mathrm{dl}$ ) had decreased cerebral blood flow, particularly in the brainstem and cerebellum, had lower mean arterial pressure and cerebral perfusion pressure, and were significantly more likely to develop postinjury apnea than control animals. ${ }^{12,16}$ These changes were observed within the first $30 \mathrm{~min}$ after TBI. When we looked specifically at the ventilatory response to injury, minute ventilation and hypercapnic respiratory drive were markedly impaired in ethanol-treated, brain-injured animals. ${ }^{16}$

Clinical studies cited herein suggest that alcohol may increase the need for prehospital ventilatory assistance in TBI patients, and laboratory data indicate that alcohol impairs respiratory drive and cerebral blood flow after TBI. Therefore, one way of explaining our findings is if cardiorespiratory and cerebrovascular changes occur immediately after serious CNSI in alcohol-intoxicated humans. In this scenario, alcohol-intoxicated CNSI patients would die a "physiological death" from apnea or hypoxia due to impaired ventilation, or decreased cerebral blood flow in the first few minutes after injury and before or during emergency medical services response. The fact that we did not find significant differences in ISS scores or AP scores between alcohol and nonalcohol groups would support this idea, because death from respiratory depression, hypoten- sion, or decreased cerebral blood flow would not be detected with these anatomical scoring systems. In addition, a standard autopsy will detect gross neuropathological changes in the brain (such as hematomas, large contusions, or brain lacerations), but will most likely not pick up more subtle changes (such as microscopic hemorrhages, cellular edema, or diffuse axonal injury). Animal models suggest that alcohol effects in TBI are due to alterations in physiological, neurochemical, and cellular function, rather than on gross anatomical changes.

Alcohol-induced physiological effects may occur through direct action on cardiovascular structures or through central neurochemical alterations. Alcohol has been shown in models of hemorrhagic shock to have a direct myocardial depressant effect that can result in decreased mean arterial pressure. ${ }^{27}$ This could secondarily lead to decreased cerebral blood flow. The neurochemical effects of alcohol are wide-ranging. Alcohol intoxication may promote free radical reactions, influence opiate and GABA receptor pathways, and alter the release and receptor binding of excitatory amino acids. ${ }^{28}$ All of these systems are felt to be involved in the early response to $\mathrm{TBI}^{29}$

A number of limitations are present in our study. We retrospectively reviewed a database using autopsy and medical examiner records. A prospective study would be better able to capture all data and would be able to define more completely injury and clinical presentation. The number of subjects in our study is smaller than the numbers reported in similar studies on MVC fatalities. An additional limitation is lack of standardization of timing of alcohol levels. Presumably, alcohol levels were drawn in the hospital ED or at autopsy in those victims who did not survive transport to the hospital. However, the database used for this study did not allow us to determine exact timing of BAC measurement.

Selection bias may have occurred in this study because BAC was not recorded in 69 of the $401(17 \%)$ original subjects. Those cases in whom BAC was not recorded had greater elapsed minutes to death than those in whom BAC was recorded, but did not have different ISS, AP scores, or $\mathrm{G}$ scores, and were not more likely to die in the first hour after the crash. The mean time to death in the nonmeasured group was $37 \mathrm{hr}$, meaning that the majority of these subjects died in the hospital. A previous study has found that only $75 \%$ of hospitalized adult trauma victims have BAC testing done in the ED. ${ }^{13}$ If it is assumed that hospital clinicians are less likely to measure BAC on MVC victims who are less likely to be intoxicated (e.g., elderly females), then a higher percentage of nontested subjects would actually be BAC-negative than in the tested group. Because nontested subjects had greater elapsed minutes to death, and were less likely to die in the first hour after the MVC, inclusion of these subjects might magnify our observed effect.

This study did not control for crash factors, such as speed, seat belt use, or vehicle deformation. Consideration 
of these factors, as shown in Waller's work, leads to a more complete understanding of the potentiating effect of alcohol in traumatic injury. We also chose to group serious upper spinal cord injury with TBI to look at all significant CNSIs. We did not separately examine TBI in terms of hemorrhagic lesions, contusions, diffuse brain injury, or brain herniation.

Interpretation of the data from this study is also made more difficult due to the fact that we do not know what type of prehospital interventions, if any, were administered to subjects. In speculating about death in the early postinjury period from apnea or cardiorespiratory arrest, it would be helpful to know if early resuscitative measures were initiated. The majority of the fatalities occurred in a region where Advanced Life Support is delivered by paramedics who are trained in airway management, endotracheal intubation, and fluid resuscitation.

This study did not differentiate acute alcohol intoxication from chronic alcohol abuse or alcoholism. Although laboratory studies have found that acute alcohol intoxication can worsen TBI, clinical studies such as ours include both novice drinkers and chronic alcoholics who are acutely intoxicated. The response to injury may be different in these two subsets of alcohol users.

Alcohol use is clearly associated with traumatic injury. In this study, alcohol-intoxicated MVC victims with CNSI died more frequently in the first hour after injury, compared with their nonintoxicated counterparts. This effect was most pronounced in subjects with isolated CNSI. These data suggest that alcohol may play a prominent role in the early response to injury in patients with CNSI.

\section{ACKNOWLEDGMENT}

The authors thank Nancy Collins for her help with manuscript preparation.

\section{REFERENCES}

1. Zink BJ, Maio RF: Alcohol use and trauma. Acad Emerg Med 1:171-173, 1994

2. MMWR: Factors potentially associated with reductions in alcoholrelated traffic fatalities-United States, 1990 and 1991. MMWR 41:893899,1992

3. Evans L, Frick MC: Alcohol's effect on fatality risk from a physical insult. J Stud Alcohol 54:441-449, 1993

4. Anda RF, Williamson DF, Remington PL: Alcohol and fatal injuries among US adults: Findings from the NHANES epidemiologic follow-up study. JAMA 260:2529-2532, 1988

5. Waller PF, Stewart JR, Hansen AR, et al: The potentiating effects of alcohol on driver injury. JAMA 256:1461-1466, 1986

6. Becker DP: Common themes in head injury, in Becker DP, Gudeman SK (eds): Textbook of Head Injury. Philadelphia, W. B. Saunders, 1989, pp 1-22

7. Miller JD: Changing patterns in acute management of head injury. J Neurol Sci 103:S33-S37, 1991
8. Flamm ES, Demopoulos HB, Seligman ML, et al: Ethanol potentiation of central nervous system trauma. J Neurosurg 46:328-335, 1977

9. Albin MS, Bunegin L: An experimental study of craniocerebral trauma during ethanol intoxication. Crit Care Med 14:841-846, 1986

10. Franco CD, Spillert CR, Spillert KR, et al: Alcohol increases mortality in murine head injury. J Natl Med Assoc 80:63-65, 1988

11. Kim HJ, Levasseur JE, Patterson JL Jr, et al: Effect of indomethacin pretreatment on acute mortality in experimental brain injury. J Neurosurg $71: 565,1989$

12. Zink BJ, Walsh RF, Feustel PJ: Effects of ethanol in traumatic brain injury. J Neurotrauma 10:275-286, 1993

13. Kraus JF, Morgenstern H, Fife D, et al: Blood alcohol tests, prevalence of involvement, and outcomes following brain injury. Am J Public Health 79:294, 1989

14. Gurney JG, Rivara FP, Mueller BA, et al: The effects of alcohol intoxication on the initial treatment and hospital course of patients with acute brain injury. J Trauma 33:709-713, 1992

15. Rönty $\mathrm{H}$, Ahonen $\mathrm{A}$, Tolonen $\mathrm{U}$, et al: Cerebral trauma and alcohol abuse. Eur J Clin Invest 23:182, 1993

16. Zink BJ, Feustel PJ: Effects of ethanol on respiratory function in traumatic brain injury. J Neurosurg 82:822, 1995

17. Fell JC, Hertz ES: The effects of blood alcohol concentration on time of death for fatal crash victims, in 34th Annual Proceedings of the Association for the Advancement of Automotive Medicine, Scottsdale, AZ, 1990, pp 69-81

18. Stewart JR: Estimating the effects over time of alcohol on injury severity, in 32nd Annual Proceedings of the Association for the Advancement of Automotive Medicine, Seattle, WA, 1988, pp 319-326

19. Dischinger PC, Soderstrom CA, Shankar BS, Cowley RA: The relationship between use of alcohol and place of death in vehicular fatalities, in 32nd Annual Proceedings of the Association for the Advancement of Automotive Medicine, Seattle, WA, 1988, pp 299-311

20. Chen B, Maio RF, Green PE, et al: Geographic variation in preventable deaths from motor vehicle crashes. J Trauma 38:228-232, 1995

21. Copes WS, Champion HR, Sacco WJ, et al: Progress in characterizing anatomic injury. J Trauma 30:1200-1207, 1990

22. Jurkovich GJ, Rivara FP, Gurney JG, et al: The effect of acute alcohol intoxication and chronic alcohol abuse on outcome from trauma. JAMA 270:51-56, 1993

23. Ward RE, Flynn TC, Miller PW, et al: Effects of ethanol ingestion on the severity and outcome of trauma. Am J Surg 144:153-157, 1982

24. Huth JF, Maier RV, Simonovitz DA, et al: Effect of acute ethanolism on the hospital course and outcome of injured automobile drivers. J Trauma 23:494-498, 1983

25. Seligman ML, Flamm ES, Goldstein BD, et al: Spectrofluorescent detection of malonaldehyde as a measure of lipid free radical damage in response to ethanol potentiation of spinal cord trauma. Lipids 12:946950,1978

26. Halt PS, Swanson RA, Faden AI: Alcohol exacerbates behavioral and neurochemical effects of rat spinal cord trauma. Arch Neurol 49: 1178-1184, 1992

27. Zink BJ, Syverud SA, Dronen SC, et al: The effect of ethanol on survival time in hemorrhagic shock in an unanesthetized swine model. Ann Emerg Med 17:15-19, 1988

28. Shanley BC, Wilce PA: Receptor changes associated with ethanolinduced brain damage. pp. 299-324, in Hunt WA, Nixon SJ (eds): Alcohol-Induced Brain Damage. Bethesda, MD, Research Monograph No. 22, U.S. Department of Health and Human Services, National Institute on Alcohol Abuse and Alcoholism, Publication No. 93-3549, 1993

29. Gentile NT, McIntosh TK: Antagonists of excitatory amino acids and endogenous opioid peptides in the treatment of experimental central nervous system injury. Ann Emerg Med 22:1028-1034, 1993 